Received: November 1, 2017

\title{
Analysis of Psychological Health Status of College Students under Examination Stress Based on EEG Neuroscience*
}

\author{
Zhaoliang $\mathrm{Li}^{1}$ \\ Jilin University
}

\begin{abstract}
Psychological stress is the main factor affecting people's health. It is very important to measure psychological stress, evaluate and intervene in human psychological health under stress. In order to evaluate the psychological health state of college students under examination stress, this study collects and analyzes EEG signal of college students with examination stress based on EEG neuroscience. The results show that genetic algorithm (GA) optimization can significantly improve the accuracy of test set, indicating that GA has strong generalization ability in the process of optimization, and it is feasible to use EEG data to analyze and evaluate the psychological health state of college students under examination stress. The EEG signal of college students with and without examination stress shows the shape of unimodal arch, and the EEG signal in the two states is multi-fractal. Different subjects have different stress and psychological states in the experimental test with big differences. College students with higher examination stress are more likely to face psychological health problems.
\end{abstract}

\section{Keywords}

Examination Stress $\bullet$ Psychological Health State $\bullet$ EEG $\bullet$ GA

\footnotetext{
*Foundation Projects: General Projects of the National Social Science Fund of China, Project number:15BSH087.

${ }^{1}$ Correspondence to: Zhaoliang Li (PhD), Psychology department, College of Philosophy and Sociology, Jilin University, Changchun 130012, China. Email: lizl@jlu.edu.cn
}

Citation: Li, Z.L. (2018). Analysis of Psychological Health Status of College Students under Examination Stress Based
on EEG Neuroscience. Educational Sciences: Theory \& $\quad$ Practice, 18(5), $1547-1557$.
http://dx.doi.org/10.12738/estp.2018.5.052


Stress is a characteristic of people's emotional state (Bayrami et al., 2012). Along with the development of social economy and science and technology, people are bearing various kinds of psychological stress in life while enjoying the convenience and the stress has become one of the factors affecting health (Metcalf \& Holmes, 2010; Hyunwoo K et al. 2018). In particular, for students who are facing many examinations, their psychological health problems under the high examination stress constantly emerge. When the stress exceeds the students' psychological load capacity, psychological diseases, such as anxiety and depression may be induced (Hesselmann et al., 2011; Beaumont \& Rugg, 1979). Therefore, the psychological health of college students under examination stress isattracting more and more attention from the society. Scholars at home and abroad also adopt various methods to evaluate and measure the psychological state of students (Schmidt et al., 2012; Brand et al., 2010). However, the traditional psychological evaluation method is subjective and can't be applied effectively (Meerwijk, Ford \& Weiss, 2015; Wessel, 2016).

The study shows that there is a close relationship between psychological stress and brain neural activity. EEG neuroscience and EEG signal acquisition technology are gradually discussed and introduced into the study of people's psychology and emotion (Osborne, Chou \& Shen, 2011; Yang \& Zhou, 2005). EEG, as a kind of electricity of human body, can be collected and processed at different parts of the scalp through non-invasive methods based on regional potential analysis of human nerve cell activity (Giorgi et al., 2013; Shim \& Kim, 2014; Bonnefond et al., 2008). In addition, EEG signal is non-invasive and not easily affected by subjective judgment with high resolution, and can be used as quantitative information in people's psychological health evaluation. Thus, it receives researchers' attention in identifying and analyzing psychological health (Saeed, Anwar, Majid \& Bhatti, 2016; Shim \& Kim, 2014). In this study, based on EEG neuroscience, the stress state induction test is carried out on college students with examination stress, and the EEG signals of the subjects are collected by EEG recorder for analyzing, pre-processing, intercepting and extracting independent component analysis (ICA). Then the psychological health state of college students under examination stress is obtained, providing theoretical basis for prevention and treatment of college students' psychological health problems.

\section{EEG Signal Acquisition and EEG Feature Extraction}

\section{EEG signal acquisition and preprocessing}

The Neuroscan EEG recorder used in the experiment has a high sampling frequency that can reach $20 \mathrm{KHz}$, and its common mode rejection ratio and input impedance are $108 \mathrm{~d}$ and $10 \mathrm{Gohm}$ respectively, which ensures the continuity of sampling information. As an advanced and complete test system in the field of EEG neuroscience, Neuroscan EEG recorder can not only accurately collect EEG signals, but also improve the accuracy of EEG signal analysis for its powerful software analysis and data processing functions (Sahoo, Mohanty \& Sahoo, 2014).

In order to ensure that the subjects are representative and random, this study selects college students with different stress states to analyze their psychological health state. 15 junior college students who take part in the postgraduate entrance exams and 15 junior college students who don't take part in the postgraduate entrance exams are selected randomly. The former have higher stress for entrance exams, while the later have lower 
stress. Among these students, the subjects with normal vision (including normal after correction) and normal body coordination ability are selected to conduct follow-up EEG test, and then their psychological health state are analyzed and evaluated. The examination stress is simulated in the form of test questions. The test questions will be read by subjects within a certain period of time before the test. The steps of EEG signal acquisition are as follows: (1) EEG signals are collected for 30-60 seconds after the subjects take a rest until the EEG signal is stable; (2) the voice signal reminds the subjects to start the test for $60 \mathrm{~s}$; (3) repeat steps (1) and (2) and record three EEG signals for each subject. The specific test flow is shown in Figure 1.

The flow chart of ERP is shown as follows

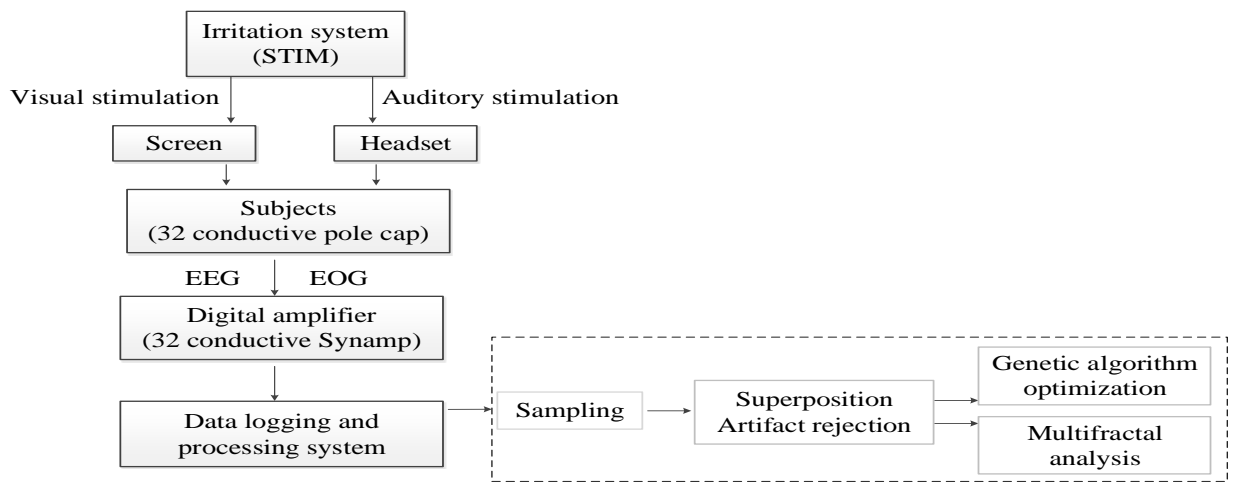

Figure 1. Flow chart of ERP experiment.

The EEG signal collected in the experiment has much background noise, interference signal and obvious fluctuation, so it needs to be pre-processed, that is, it is intercepted, filtered and removed the horizontal EOG effectively. After that, the EEG signal is subjected to sampling frequency conversion processing. The frequency of the signal is converted to $128 \mathrm{~Hz}$ and stored in an Excel file, and is intercepted as an EEG sample set processed by ICA to remove the signal artefacts.

\section{EEG feature extraction}

This study takes C4 electrode as the object of study, extracts three eigenvectors of Kc complexity, C0 complexity and approximate entropy (ApEn), and uses back propagation (BP) neural network classifier to identify the stress state of subjects, which provides theoretical and data support for evaluating their psychological health state.

\section{Kc complexity}

To solve the complexity of EEG signals, the original EEG signals need to be coarsened to make the continuous EEG signals become binary signals with limited time series elements. The complexity calculation is realized by means of Matlab program in the steps: the data are imported, the average value is calculated, the numerical value is compared and the data are saved (the data which are greater than the average value are saved as 1 and stored in the newly generated matrix, the data which are smaller than the average value are saved as 0 
and input into the newly generated sequence). Then the L-Z algorithm programming is carried out for the calculation of Kc complexity.

\section{CO complexity}

$\mathrm{C} 0$ complexity represents the irregular degree of the series and reflects the rate at which a new pattern occurs with the increasing length of the time series. The C0 complexity is obtained by fast calculation of Fourier transform. As shown in Formula 1, $\mathrm{C} 0$ is the ratio of the area of the region bounded by $\left|s(t)-s^{\prime}(t)\right|$ and $s(t)$. The larger the randomness of the time series is, the larger the value of $\mathrm{C} 0$ complexity is. If the time series is periodic, $\mathrm{C} 0$ complexity tends to be 0 with the increase of the length of series. If the time serie is constant, $\mathrm{C} 0$ complexity is 0 .

$$
C 0=\frac{\sum_{t=1}^{N}\left|s(t)-s^{\prime}(t)\right|}{\sum_{t=1}^{N}|s(t)|}
$$

Where, $s(t)$ is original time series, $s^{\prime}(t)$ is time series after Fourier transform.

\section{ApEn}

ApEn is used to measure the complexity of time series, which reflects the self-similarity of EEG signal in time series. The more complex the time series of EEG signal is, the larger the ApEn value is. Similarly, the simpler the time series is, the smaller the ApEn value is.

\section{BP neural network and GA}

BP neural network can be trained by error back propagation algorithm. The topology of BP neural network model includes input layer, hidden layer and output layer, as shown in Figure 2. GA can simulate natural selection and genetic mechanism to perform model calculation so as to search for the optimal solution of the data set. In this study, GA is used to optimize support vector machine (SVM). Neural network and GA are used to optimize weights and thresholds. The detailed flow is shown in Figure 3.

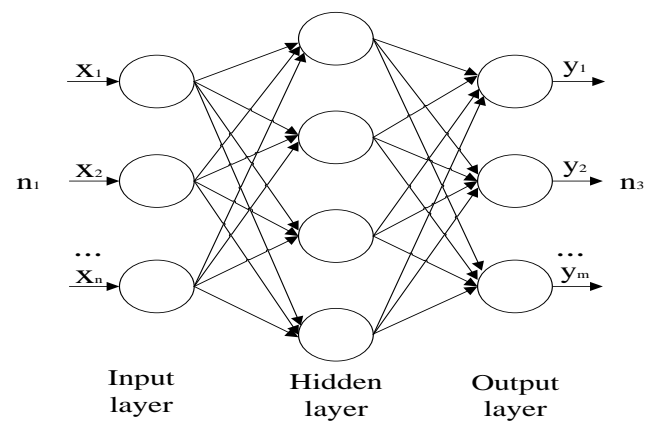

Figure 2. BP neural network structure model. 


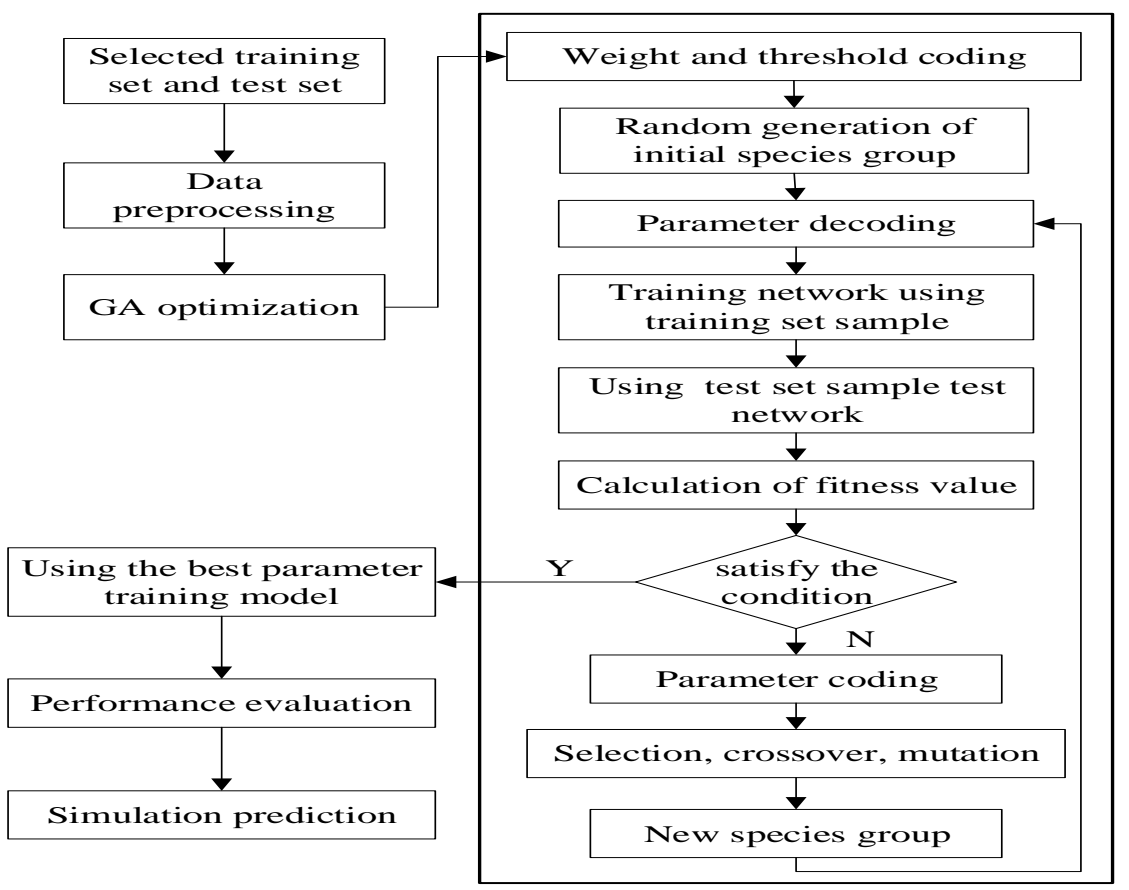

Figure 3. GA-BP neural network flow chart.

\section{Experimental Result and Analysis}

\section{Optimization of EEG data by genetic algorithm}

In each stimulation test, 66 samples are randomly selected as the training set, and 24 samples are used as the test set. In this study, the number of samples in the training set and the test set is about $3: 1$. The BP neural network GA and SVM GA are simulated and tested for ten times before and after optimization. Table 1 shows the accuracy of the test set identification of GA-SVM optimized by GA and SVM without optimization. It can be seen that the average accuracy of the test set of SVM before and after the GA optimization is $70.86 \%$ and $80.83 \%$ respectively. The average accuracy of the test set after the GA optimization increases by $9.97 \%$, indicating that GA can improve the classification identification accuracy of the SVM classifier and optimizes the identification result. It also shows that it is feasible to use EEG data to analyze and evaluate the psychological health state of college students under examination stress.

Table 1

10 SVM and GA-SVM Tests Set Accuracy (\%)

\begin{tabular}{lccccc}
\hline SVM & 73.26 & 78.91 & 73.88 & 73.94 & 68.57 \\
GA-SVM & 78.61 & 82.26 & 78.23 & 86.99 & 77.26 \\
\hline SVM & 79.26 & 60.77 & 65.32 & 69.47 & 65.32 \\
GA-SVM & 92.10 & 78.15 & 69.68 & 80.18 & 84.61 \\
\hline
\end{tabular}




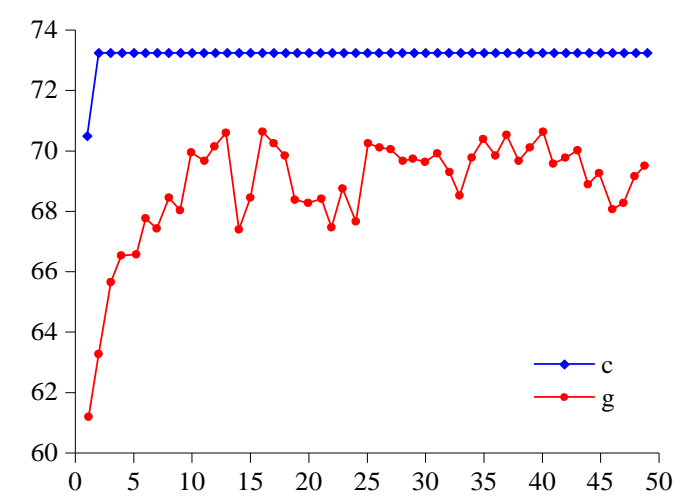

Figure 4. Optimization process of c, g in one experiment.

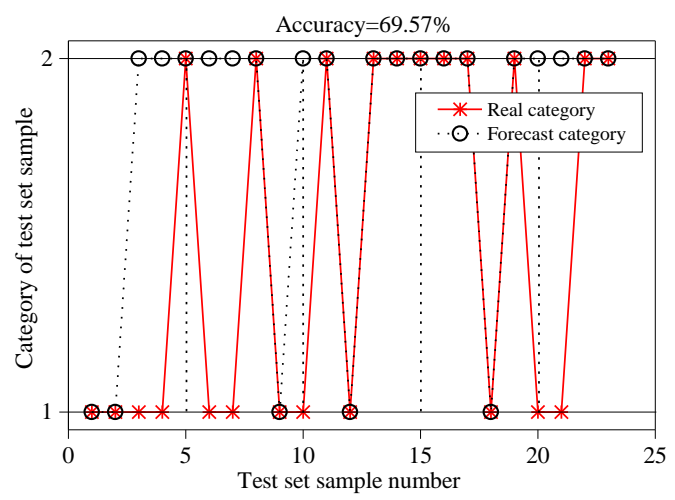

Figure 5. SVM test set accuracy.

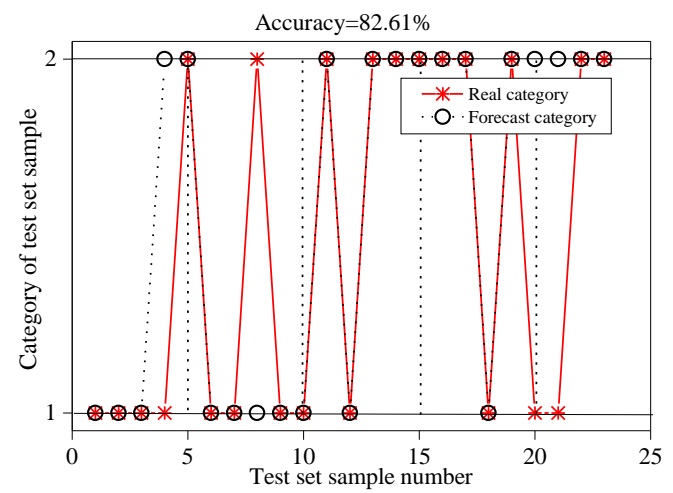

Figure 6. GA-SVM test set accuracy. 
Figure 4 shows the optimization process of the error penalty factor $\mathrm{c}$ and the kernel function $\mathrm{g}$ of the RBF kernel function in the stimulation test. In one simulation experiment, the best quality of the error penalty factor $\mathrm{c}$ and the kernel function $\mathrm{g}$ is 5.8542 and 8.7218 respectively. The simulation result of the classification accuracy of the default SVM test set is shown in Figure 5 while that of SVM optimized by GA is shown in Figure 6. It can be found from comparison that the accuracy of SVM before and after GA optimization is $69.57 \%$ and $82.61 \%$ respectively. GA optimization improves the classification accuracy of SVM by more than $13 \%$.

The classification accuracy of BP neural network optimized by genetic algorithm (GA-BP) is further studied. The accuracy of BP neural network test samples before and after GA optimization for 10 times is shown in Table 2. It can be seen that the average accuracy of BP neural network test before and after GA optimization is $69.47 \%$ and $81.64 \%$ respectively. After GA optimization, the average accuracy of BP neural network test set is improved by more than $12 \%$, indicating that GA can optimize the accuracy of BP neural network simulation test set. The result also shows that EEG data can be used to evaluate the psychological health state of college students under examination stress.

Table 2

\begin{tabular}{llllll}
10 BP and GA-BP Tests Set Accuracy & \\
\hline BP & 73.81 & 65.12 & 78.16 & 82.51 & 60.77 \\
GA-BP & 81.61 & 73.81 & 91.20 & 82.51 & 73.81 \\
\hline BP & 60.79 & 60.65 & 69.48 & 60.86 & 82.51 \\
GA-BP & 82.51 & 73.81 & 78.16 & 91.20 & 86.86 \\
\hline
\end{tabular}

\section{Multi-fractal analysis of EEG under stress}

According to the study, EEG signals have obvious fractal features, that is, the EEG signals have no fixed feature length, but have self-similar pattern structure. When the shapes and amplitudes of EEG signals of the two groups are similar, they can't be effectively divided in time domain, so the fractal characteristics of EEG signals can be analyzed by the singular spectral curves of singular intensity $\alpha$ and $f(\alpha)$. First, based on the EEG signals of the two states of resting and opening the eyes and resting and closing the eyes, fractal method is used to obtain the multi-fractal spectra of the EEG signals under the two states, as shown in Figure 7. It can be seen that the width and amplitude of the singular spectrum are not the same under the two different states of EEG signals, which can be distinguished from each other.

Based on the multi-fractal theory, the multi-fractal spectrum of EEG under stress and non-stress state is obtained. As shown in Figure 8, EEG signals under both examination and non-stress state show unimodal arch, showing that EEG signals in the two states are multi-fractal signals. According to the analysis results, 14 groups of EEG data without stress and 20 groups of EEG data with stress are selected for further analysis. The multifractal spectrum analysis results of EEG data under the two states are shown in Table 3 and Table 4. According to the statistical results, it can be found that different subjects have different stress and psychological state in the test, and there are great differences among individuals. The college students with higher examination stress are more likely to face psychological health problems. Therefore, the average value of the other fractal spectrum widths after the maximum value and the minimum value of the singular spectrum width $\Delta \alpha$ are removed. As shown in Table 5, the EEG signal $\triangle \alpha$ under examination stress is larger than that under no examination stress, 
so $\triangle \alpha$ can be used to distinguish the EEG signals under different examination stress state, as well as to analyze and evaluate the psychological health state of college students under examination stress.

Table 3

Statistical Analysis of Multi-fractal Spectrum of Stress EEG Signals

\begin{tabular}{cccccccc}
\hline $\begin{array}{l}\text { Group } \\
\text { number }\end{array}$ & $\begin{array}{c}\alpha \\
\text { maximum }\end{array}$ & $\begin{array}{c}\alpha \\
\text { minimum }\end{array}$ & $\Delta \alpha$ & $\begin{array}{c}\text { Group } \\
\text { number }\end{array}$ & $\begin{array}{c}\alpha \\
\text { maximum }\end{array}$ & $\begin{array}{c}\alpha \\
\text { minimum }\end{array}$ & $\Delta \alpha$ \\
\hline 3 & 1.57 & 0.39 & 1.18 & 22 & 1.47 & 0.55 & 0.92 \\
4 & 0.71 & 0.28 & 0.43 & 24 & 1.71 & 0.52 & 1.19 \\
7 & 1.27 & 0.34 & 0.93 & 25 & 1.32 & 0.47 & 0.85 \\
9 & 0.61 & 0.27 & 0.35 & 26 & 0.86 & 0.58 & 0.28 \\
10 & 0.72 & 0.36 & 0.36 & 28 & 1.17 & 0.43 & 0.74 \\
13 & 0.79 & 0.32 & 0.47 & 30 & 1.10 & 0.41 & 0.69 \\
14 & 0.88 & 0.31 & 0.57 & 31 & 0.73 & 0.35 & 0.38 \\
17 & 0.89 & 0.39 & 0.50 & 33 & 0.96 & 0.41 & 0.55 \\
18 & 0.85 & 0.51 & 0.34 & 36 & 1.10 & 0.38 & 0.72 \\
21 & 1.25 & 0.41 & 0.84 & 37 & 1.27 & 0.34 & 0.93 \\
\hline
\end{tabular}

Table 4

Multi-fractal Spectrum of Stress-Free EEG Signal

\begin{tabular}{cccccccc}
\hline $\begin{array}{l}\text { Group } \\
\text { number }\end{array}$ & $\begin{array}{c}\alpha \\
\text { maximum }\end{array}$ & $\begin{array}{c}\alpha \\
\text { minimum }\end{array}$ & $\triangle \alpha$ & $\begin{array}{c}\text { Group } \\
\text { number }\end{array}$ & $\begin{array}{c}\alpha \\
\text { maximum }\end{array}$ & $\begin{array}{c}\alpha \\
\text { minimum }\end{array}$ & $\Delta \alpha$ \\
\hline 1 & 0.92 & 0.30 & 0.62 & 21 & 0.84 & 0.16 & 0.68 \\
2 & 0.63 & 0.44 & 0.19 & 37 & 0.97 & 0.24 & 0.73 \\
4 & 0.89 & 0.42 & 0.47 & 40 & 0.83 & 0.45 & 0.38 \\
6 & 0.94 & 0.21 & 0.73 & 41 & 0.63 & 0.27 & 0.36 \\
11 & 0.80 & 0.50 & 0.30 & 44 & 0.74 & 0.34 & 0.40 \\
15 & 1.09 & 0.41 & 0.69 & 48 & 0.91 & 0.33 & 0.58 \\
20 & 1.48 & 0.31 & 1.17 & 49 & 1.09 & 0.34 & 0.75 \\
\hline
\end{tabular}

Table 5

Analysis and Comparison of the Range of Singular Spectrum of EEG with or without Stress

\begin{tabular}{lccc}
\hline & Maximum $\triangle \alpha$ & Minimum $\triangle \alpha$ & Mean $\triangle \alpha$ \\
\hline without pressure & 1.17 & 0.19 & 0.56 \\
Under pressure & 1.19 & 0.28 & 0.65 \\
\hline
\end{tabular}

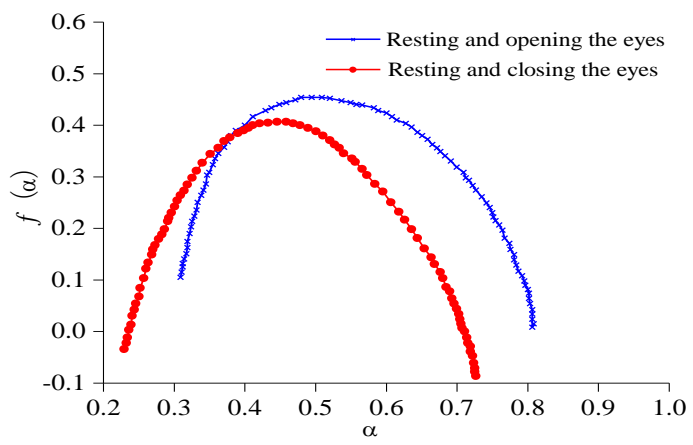

Figure 7. Multi-fractal spectrum of eyes opening and closing. 


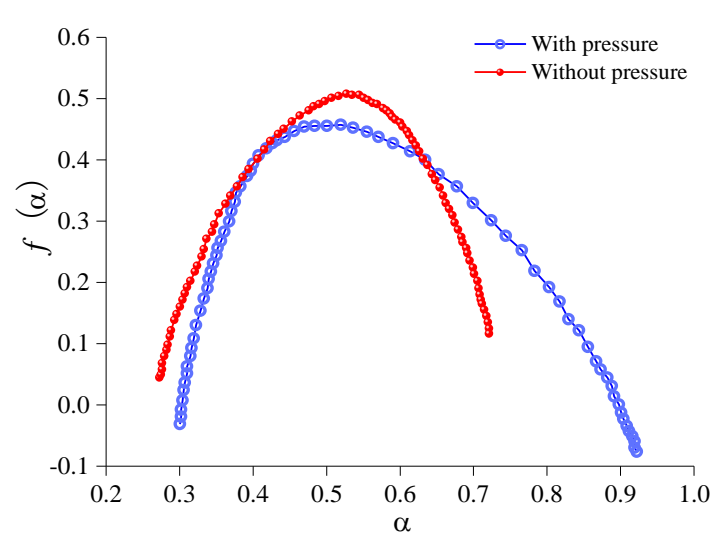

Figure 8. Multi-fractal spectrum of electroencephalogram with stress and without stress.

\section{Conclusion}

Based on the research of EEG neuroscience, this study analyzes the psychological health state of college students under examination stress by using multi-fractal theory and support vector optimized by GA and BP neural network. Main conclusions have been drawn as follows:

(1) According to the characteristics of EEG signals of college students under examination stress, the eigenvectors of Kc complexity, $\mathrm{C} 0$ complexity and ApEn are selected to analyze and calculate the EEG data.

(2) After GA optimization, the average accuracy of test set of SVM simulation experiment is improved by $9.97 \%$ and the accuracy of BP neural network test set is improved by more than $12 \%$ on average, which indicates that GA has strong generalization ability in the process of optimization, and it is feasible to use EEG data to analyze and evaluate the psychological health state of college students under examination stress.

(3) The EEG signals of the students under the state with stress and without stress show the shape of unimodal arch, which indicates that the EEG signals under the two states are multi-fractal signals. In the experiment, different subjects have different stress and psychological states, and there are great differences among individuals. College students with higher examination stress are more likely to face psychological health problems.

\section{References}

Bayrami, M., Hashemi, T., Malekirad, A. A., Ashayeri, H., Faraji, F., \& Abdollahi, M. (2012). Electroencephalogram, cognitive state, psychological disorders, clinical symptom, and oxidative stress in horticulture farmers exposed to organophosphate pesticides. Toxicology \& Industrial Health, 28(1), 90-96. http://dx.doi.org/10.1177/0748233711407243 
Beaumont, J. G., \& M.D. Rugg. (1979). The specificity of intrahemispheric EEG alpha coherence asymmetry related to psychological task. Biological Psychology, 9(4), 237. http://dx.doi.org/10.1016/03010511(79)90025-5

Bonnefond, A., Rohmer, O., Engasser, O., Hoeft, A., Eschenlauer, R., \& Muzet, A., et al. (2008). Effects of age and task complexity on EEG and performance in relation to psychological states. Journal of Psychophysiology, 22(2), 100-109. http://dx.doi.org/10.1027/0269-8803.22.2.100

Brand, S., Gerber, M., Beck, J., Hatzinger, M., Pã1/4Hse, U., \& Holsboer-Trachsler, E. (2010). Exercising, sleepEEG patterns, and psychological functioning are related among adolescents. World Journal of Biological Psychiatry, 11(2), 129-140. http://dx.doi.org/10.3109/15622970903522501

Giorgi, F. S., Pizzanelli, C., Pelliccia, V., Coscio, E. D., Maestri, M., \& Guida, M. (2013). A clinical-EEG study of sleepiness and psychological symptoms in pharmacoresistant epilepsy patients treated with lacosamide. Epilepsy Research and Treatment, (6), 593149. http://dx.doi.org/10.1155/2013/593149

Hesselmann, G., Flandin, G., \& Dehaene, S. (2011). Probing the cortical network underlying the psychological refractory period: a combined EEG-fmri study. Neuroimage, 56(3), 1608. http://dx.doi.org/10.1016/j.neuroimage.2011.03.017

Hyunwoo K., Seyong J. (2018), Effects of competition anxiety on self-confidence in soccer players: Modulation effects of home and away games, Journal of Men's Health, 14(3), e62-e68; DOI: 10.22374/18756859.14.3.9

Konareva, I. N. (2011). Modulation of high-frequency EEG rhythms under conditions of the activation reaction: dependence on psychological characteristics of personality. Neurophysiology, 43(1), 42-52. http://dx.doi.org/10.1007/s11062-011-9184-6

Meerwijk, E. L., Ford, J. M., \& Weiss, S. J. (2015). Resting-state EEG delta power is associated with psychological pain in adultswith a history of depression. Biological Psychology, 105, 106-114. http://dx.doi.org/10.1016/j.biopsycho.2015.01.003

Metcalf, D. R., \& Holmes, J. H. (2010). EEG, psychological, and neurological alterations in humans with organophosphorus exposure*. Annals of the New York Academy of Sciences, 160(1), 357-365. http://dx.doi.org/10.1111/j.1749-6632.1969.tb15857.x

Osborne, P. G., Chou, T. S., \& Shen, T. W. (2011). Characterization of the psychological, physiological and EEG profile of acute betel quid intoxication in naïve subjects. Plos One, 6(8), e23874. http://dx.doi.org/10.1371/journal.pone.0023874

Saeed, S. M. U., Anwar, S. M., Majid, M., \& Bhatti, A. M. (2016). Psychological stress measurement using low cost single channel EEG headset. IEEE International Symposium on Signal Processing and Information Technology, 581-585. http://dx.doi.org/10.1109/isspit.2015.7394404

Sahoo, S., Mohanty, S., \& Sahoo, T. (2014). Association between psychology and technical education by EEG. Advance Computing Conference, 1315-1321. http://dx.doi.org/10.1109/iadcc.2014.6779517

Schmidt, S., Naranjo, J. R., Brenneisen, C., Gundlach, J., Schultz, C., \& Kaube, H., et al. (2012). Pain ratings, psychological functioning and quantitative EEG in a controlled study of chronic back pain patients. Plos One, 7(3), e31138. http://dx.doi.org/10.1371/journal.pone.0031138 
Shim, J. M., \& Kim, S. J. (2014). Effects of manual lymph drainage of the neck on EEG in subjects with psychological stress. Journal of Physical Therapy Science, 26(1), 127-129. http://dx.doi.org/10.1589/jpts.26.127

Shim, J. M., \& Kim, S. J. (2014). Manual lymph drainage attenuates frontal EEG asymmetry in subjects with psychological stress: a preliminary study. Journal of Physical Therapy Science, 26(4), 529-531. http://dx.doi.org/10.1589/jpts.26.529

Wessel, J. R. (2016). Testing multiple psychological processes for common neural mechanisms using EEG and independent component analysis. Brain Topography, 31(Suppl D), 1-11. http://dx.doi.org/10.1007/s10548016-0483-5

Yang, Y., \& Zhou, J. (2005). Recognition and Analyses of EEG\&ERP Signals Related to Emotion: From the Perspective of Psychology. First International Conference on Neural Interface and Control, Proceedings, 96-99. http://dx.doi.org/10.1109/icnic.2005.1499851 